\title{
Congressos \\ e Conferências
}

Maria Regina Tavares ${ }^{a}$

ABRIL 1990

9-11 Southampton Analitical Methods of Sea Water Analysis (GB)

16-20 Wiesbaden 2nd European Inorganic Chemistry Seminar (RFA)

\section{MAIO 1990}

\section{6-9 Alicante} (E)

15-18 Ghent (B)

? Gregynog (GB)

20-25 Kyoto (J)

20-25 Jerusalém (Israel)

22-24 Lisbon (P)

\section{JUNHO 1990}

$\begin{array}{ll}\mathbf{5 - 8} & \text { Espoo } \\ & \text { (Finlândia) }\end{array}$

5-9 Strasbourg 1st International Congress on Ethnopharma(F) cology

10-14 Visby (Suécia)

25-30 Umea (Suécia)

\section{JULHO 1990}
1-1 Tubingen (RFA)
3-5 Galway (Irlanda)
8-13 Montreal (Canadá)
8-14 Copenhague (D)
International Conference on Synthetic Metals' 90
1,3-Dipoles in Organic Synthesis
33rd IUPAC International Symposium on Macromolecules
5th European Congress of Biotechnology

International Symposium on the Analysis of Inhibitors of Ensymes of Plants

9th International Simposium on Carotenoids

19th International Symposium on Polymer Networks

6th European Symposium on Organic Micropollutants in the Aquatic Environment

International Symposium on Trace Elements in Health and Disease

Chemical and Biological Aspects of Nicotine and Related Alkaloids

Statistical Design of Chemical Experiments

13th IUPAC Symposium on Photochemistry
23-25 Brno (Checosl.)

23-27 Helsínquia (Finlândia)

30-3 Torun (P)

\section{AGOSTO 1990}

\section{? $\quad$ Stockolm} (Suécia) Anabolizing and Doping Agents in Biosamples 
9-13 Paris

(F)

10-13 Basel

(S)

11-14 Kode

(J)

\section{OUTUBRO 1990}

1-4 Avignon

(F)

2-5 Poitiers (F)

\section{FEVEREIRO 1991}

10-15 Melbourne (Austrália)
International Symposium on Mechamisms and Kinetics of Polymerization Reactions: Their Use in Polymer Synthesis

1st IUPAC Workshop on Safety in Chemical Production

12th International Symposium on Capillary Chromatografy

Chimie Théorique et Synthèse Organique

2nd International Symposium CNRS on Heterogeneous Catalysis and Fine Chemicals

International Conference Polymer 91
ABRIL 1991

15-19 Baden-Baden 2nd International Symposium "Organic (RFA) Chemistry": Technological Perspectives

JUNHO 1991

16-21 Karlsruhe World Congress IV on Chemical Engineering (RFA)

AGOSTO 1991
1-1 Bochum (RFA)

17-22 Budapest 33rd IUPAC Congress

(H)

25-29 Netherlands 6th International Symposium on Organometallic (H) Chemistry Directed Towards Organic Synthesis

26-30 Amsterdam (H) 12th International Mass Spectrometry Conference 13th International Fluor-Symposium

(H) rence

\section{NÃO ATRASE}

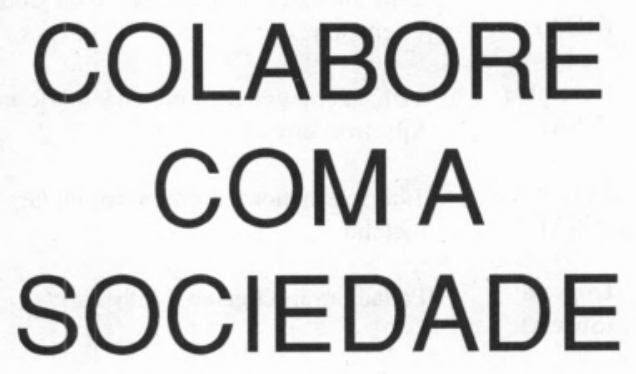

O

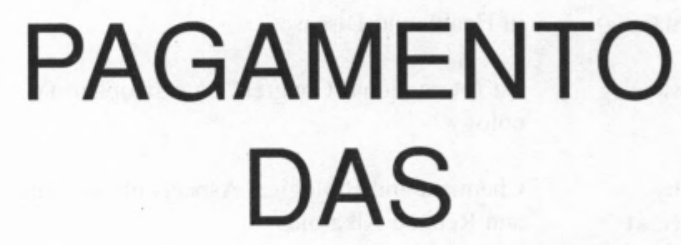

SUAS QUOTAS 